\title{
Der Einsatz von Video in Forschung und Lehre hat ein neues Niveau erreicht
}

\author{
Michel Knigge $\cdot$ Jens Siemon
}

Videoaufnahmen sind ein mächtiges Werkzeug für Forschung und Lehre. Obwohl diese Aussage schon seit der Entwicklung von Videoaufnahmegeräten gilt, kann man doch einen neuen Trend zur Nutzung dieser Technik ausmachen. Der Einsatz von Video ist in jüngster Zeit zunehmend beliebter und vor allem vielfältiger geworden. Dies liegt sicherlich nicht zuletzt daran, dass die Technologie sich rasant entwickelt hat und viele technische Hürden inzwischen sehr niedrigschwellig geworden sind. Kleine, handliche, quasi vollautomatische Kameras ermöglichen eine sehr gute Bild- und Tonqualität auch für Personen, die nicht professionell im Filmgeschäft sind. Digitale Speichermedien in kleiner Größe mit großem Fassungsvermögen erlauben die Aufnahme von nahezu unbegrenzten Mengen an Videomaterial. Und zum Abspielen reichen ein Notebook, ein Videobeamer und ein paar kleine Lautsprecher, so dass seit einigen Jahren Aufnahme und Wiedergabe von Videos quasi überall mit vertretbarem Aufwand (im Hinblick auf Zeit und Geld) möglich sind. Vermutlich ist dies ein wichtiger Grund, warum in Forschung und Lehre zunehmend Videoaufnahmen zum Einsatz kommen.

Doch würden die technischen Erleichterungen kaum zu einer größeren Verbreitung einer videobasierten Methodik führen, wenn diese nicht auch inhaltlich in vielerlei Hinsicht gewinnversprechend wäre. Der Einsatz von Video in der Forschung erlaubt es, komplexe pädagogische und psychologische Prozesse - wie beispielsweise Gruppendynamiken - mit einer hohen intersubjektiven Nachvollziehbarkeit zu untersuchen (vgl. Corsten et al. 2010; Derry 2007). Wenn die Videoaufnahmen einmal gemacht sind, können sie beliebig oft wieder gesichtet und analysiert werden. Sämtliche Schlussfolgerungen, die gezogen werden, sind anhand von Daten überprüfbar, die gegenüber ihrem realen Ursprung verhältnismäßig wenig reduziert bzw. verändert wurden. Dabei können Videodaten qualitativ und quantitativ ausgewertet werden und mit Videomaterial sind grundlagen- und anwendungsorientierte Forschungsarbeiten möglich. Aber nicht nur in

\author{
Online publiziert: 10.07 .2013 \\ (C) Springer Fachmedien Wiesbaden 2013 \\ Dr. M. Knigge $(\bowtie) \cdot$ Prof. Dr. J. Siemon \\ Universität Hamburg, Hamburg, Deutschland \\ E-Mail: michel.knigge@uni-hamburg.de; michel.knigge@gmail.com \\ Prof. Dr. J. Siemon \\ E-Mail: jens.siemon@uni-hamburg.de
}


der Forschung erscheint der Einsatz von Videos lohnenswert. Videos können auch zur Reflexion eingesetzt werden. Videoaufzeichnungen erlauben einen äußeren Blick auf das eigene Handeln. Wie beispielsweise Gärtner (2007) zeigen konnte, profitierten nicht nur die teilnehmenden Lehrkräfte von einem videobasierten Qualitätszirkel sondern auch ihre Schülerinnen und Schüler. Um der - unter anderem von Hattie (2009) - betonten Bedeutsamkeit von Feedback für alle am pädagogischen Prozess beteiligten Personen Genüge zu tun, ist Video ein hervorragendes Werkzeug. Nicht zuletzt können Videoaufnahmen als Lehrmittel genutzt werden. Mit ihnen können Beispiele von Best Practice so vermittelt werden, dass eine Reproduktion leichter fällt. Der Transfer von Theorien in die Praxis kann durch den Einsatz von Video erleichtert werden. Damit bietet diese Methode auch für in der Ausbildung befindliche angehende Lehrkräfte eine gute Möglichkeit, systematisch Stärken und Schwächen von normalen Unterrichtssituationen zu analysieren.

Im vorliegenden Themenheft werden verschieden Formen der Einsatzmöglichkeiten von Video beschrieben und untersucht. Dabei zeigen sich an vielen Stellen bedeutsame Innovationen, die zur Nachahmung und Erweiterung einladen. Der erste Beitrag von Kristin Bührig und Joana Duarte beschäftigt sich mit Mehrsprachigkeit im Fachunterricht. Die Autorinnen greifen dabei auf Daten zurück, die im Hamburger Landesexzellenzcluster LiMA mit einer innovativen Technik gewonnen wurden, bei der für alle Schülerinnen und Schüler individuelle Tonspuren aufgezeichnet werden. Anhand von Fallbeispielen im Rahmen von Partnerarbeiten zeigen sie Beispiele eines produktiven Einsatzes von Mehrsprachigkeit im Fachunterricht, die nur auf Grund der neuen Methodik möglich geworden sind. Mit der gleichen Videotechnologie untersuchen Michel Knigge, Jens Siemon, Vibeke Nordstrand und Claudia Stolp im zweiten Beitrag auch Interaktionen in Dyaden. Individuelle Zielorientierungen und kognitive Grundfähigkeiten werden mit dem Interaktionsverhalten in den Zweiergruppen regressionsanalytisch in Beziehung gesetzt. Dabei kann gezeigt werden, dass die Interaktionen systematisch mit individuellen und dyadischen Zielorientierungen und kognitiven Grundfähigkeiten variieren. Der dritte Beitrag von Marten Clausen, Heike Wegner und Jörg Weingarten zeigt den praxisnahen Fall des Einsatzes von Video zur Evaluation des Unterrichts. Am Beispiel einer Internatsschule wird gezeigt, wie Videografie genutzt werden kann, um Stärken und Schwächen auf der Prozessebene zu identifizieren. Die Autor/innen nutzen Daten und Instrumente aus der TIMSS-Videostudie als Referenz, wodurch die Ergebnisse über eine reflexive Komponente hinaus eine quantifizierbare Verortung auf Qualitätsskalen für Unterricht ermöglichen. Im vierten Beitrag wird von Kristin Bührig und Carmen Spiegel eine traditionelle Stärke von Videostudien aufgegriffen, indem sie diskursanalytisch einen zentralen Mikroaspekt von Unterricht untersuchen. Im Rahmen einer Analyse einer Argumentationsübung zeigen sie exemplarisch Schwierigkeiten und Potenziale zur Optimierung des Handelns von Lehrkräften. Dabei nehmen sie den Einsatz von Fragen von Seiten der Lehrkraft besonders in den Blick und verdeutlichen, welche Folgen ihr Einsatz mit sich bringen kann. Abgerundet wird das Themenheft mit dem fünften Beitrag von Kathleen Stürmer und Tina Seidel zum Einsatz von Video in der Ausbildung von Lehrerinnen und Lehrern. In dem von Ihnen beschriebenen Theorie-Praxis-kombinierten Ausbildungsprogramm wird Video in zweierlei Hinsicht genutzt: Einerseits werden Videobeispiele genutzt, um die Lehre anzureichern und praxisnäher zu gestalten. Ande- 
rerseits wird das videobasierte Tool Observer genutzt, um Lernfortschritte bei den Studierenden zu überprüfen.

Die Beiträge in diesem Themenheft zeigen das große Innovationspotenzial von Video in Forschung und Lehre. Es wird deutlich, dass hier ein neues Niveau erreicht worden ist, sowohl was die Ausgestaltung der Methode selbst als auch was ihren Einsatzbereich angeht. Es zeigt sich aber auch, wie viele Möglichkeiten für die weitere Entwicklung noch ungenutzt bestehen und welche Chancen diese für neue wissenschaftliche Erkenntnisse und die Verbesserung von universitärer Lehre, Schule und Unterricht bereithalten könnten. In diesem Sinne sind wir uns sicher, dass wir mit dem vorliegenden Themenheft einen inspirierenden Beitrag zur weiteren Beförderung zum Einsatz von Video in Forschung und Lehre geleistet haben und wünschen Ihnen eine spannende Lektüre.

\section{Literatur}

Corsten, M., Krug, M., \& Moritz, C. (2010). Videographie praktizieren. Herangehensweisen, Möglichkeiten und Grenzen. Heidelberg: Springer VS.

Derry, S. J. (Hrsg.). (2007). Guidelines for Video Research in Education. Recommendations from an Expert Panel. Data Research and Development Center: University of Chicago. http://drdc. uchicago.edu/what/video-research-guidelines.pdf. Zugegriffen: 10. Juni 2013.

Gärtner, H. (2007). Unterrichtsmonitoring - Evaluation eines videobasierten Qualitätszirkels zur Unterrichtsentwicklung. Münster: Waxmann.

Hattie, J. A. C. (2009). Visible learning. A synthesis of over 800 meta-analyses relating to achievement. London: Routledge. 